\title{
Clinical Study \\ Effect of Admission Time on the Outcomes of Liver Cirrhosis with Acute Upper Gastrointestinal Bleeding: Regular Hours versus Off-Hours Admission
}

\author{
Yingying Li, ${ }^{1,2}$ Bing Han $\mathbb{D}^{1,},{ }^{1,2}$ Hongyu Li $\mathbb{D},{ }^{1}$ Tingxue Song, ${ }^{1,3}$ \\ Wenchun Bao, ${ }^{1,3}$ Ran Wang, ${ }^{1}$ Zhaohui Bai, ${ }^{1,4}$ Kexin Zheng, ${ }^{1,2}$ Qianqian Li,,5 \\ Xiaozhong Guo ${ }^{1}{ }^{1}$ and Xingshun Qi ${ }^{1}$ \\ ${ }^{1}$ Department of Gastroenterology, General Hospital of Shenyang Military Area, Shenyang, 110840, China \\ ${ }^{2}$ Postgraduate College, Jinzhou Medical University, Jinzhou 121001, China \\ ${ }^{3}$ Postgraduate College, Liaoning University of Traditional Chinese Medicine, Shenyang, 110840, China \\ ${ }^{4}$ Postgraduate College, Shenyang Pharmaceutical University, Shenyang 110840, China \\ ${ }^{5}$ Postgraduate College, Dalian Medical University, Dalian 116044, China

\begin{abstract}
Correspondence should be addressed to Hongyu Li; 13309887041@163.com, Xiaozhong Guo; guo_xiao_zhong@126.com,
\end{abstract} \\ and Xingshun Qi; xingshunqi@126.com
}

Received 1 August 2018; Revised 29 October 2018; Accepted 7 November 2018; Published 29 November 2018

Guest Editor: Eduardo Garcia Vilela

Copyright (C) 2018 Yingying Li et al. This is an open access article distributed under the Creative Commons Attribution License, which permits unrestricted use, distribution, and reproduction in any medium, provided the original work is properly cited.

Background and Aims. Acute upper gastrointestinal bleeding (AUGIB) is a lethal complication of liver cirrhosis. We aimed to compare the outcomes of patients with liver cirrhosis and AUGIB who were admitted to hospital on regular hours and off-hours. Methods. This retrospective study screened all cirrhotic patients with AUGIB who were admitted to our hospital from January 2010 to June 2014 for the test cohort and from December 2014 to March 2018 for the validation cohort. A 1:1 propensity score matching analysis was performed to adjust the Child-Pugh and MELD scores. In-hospital mortality, 5-day rebleeding rate, length of stay, and total payment were primary outcomes. Results. Overall, 826 and 173 patients with liver cirrhosis and AUGIB were included in the test and validation cohorts, respectively. After propensity score matching, 226 and 40 patients were included in the test and validation cohorts, respectively. The overall analysis of the test cohort found significantly higher Child-Pugh score $(\mathrm{P}=0.006), 5$-day rebleeding rate (18.69\% versus $10.72 \%, P=0.001)$, and total payment ( $¥ 25,906.83$ versus $¥ 22,017.42, \mathrm{P}<0.001)$ in patients admitted on off-hours. By contrast, the overall analysis of the validation cohort did not find any difference in Child-Pugh score, 5-day rebleeding, in-hospital mortality, length of stay, or hospital payment between patients admitted on regular hours and off-hours. Similarly, the propensity score matching analyses of both test and validation cohorts found no difference in these primary outcomes between the two groups. Conclusions. Off-hours admission might not be negatively associated with the outcomes of patients with liver cirrhosis and AUGIB.

\section{Introduction}

Liver cirrhosis is the 13th major cause of death worldwide. Acute upper gastrointestinal bleeding (AUGIB) is a frequent medical emergency with a high incidence of 45-172/100,000 each year in the general population and is a lethal complication of liver cirrhosis leading to an in-hospital mortality of $10 \%[1,2]$. Due to the acute performance of AUGIB itself, early diagnosis and timely management are needed. Notably, there are general shortage of staff, a potentially lower professional level of staff, and delayed use of endoscopy during weekends and holidays, which may lead to worse outcomes $[3,4]$. Previous studies evaluated the effect of admission time on the outcomes of patients with AUGIB, but their findings were inconsistent. Some authors supported the "weekend effect" that patients admitted during weekends had worse outcomes [5-9], such as higher mortality and rebleeding rate, longer length of stay, and increased cost. On the contrary, others suggested no significant difference in the mortality between patients admitted during weekends and weekdays [10-15]. 
A meta-analysis [16] reported that off-hours admission was significantly associated with an increased mortality and less timely endoscopy in patients with nonvariceal bleeding but not those with variceal bleeding. More recently, another meta-analysis [17] also had similar results. However, there were some limitations in previous studies.

First, meta-analyses have shown that geographical variation leads to different weekend effect on outcomes [16, 17]. A study, which included 571 patients suspected with upper gastrointestinal bleeding (UGIB) from 8 participating hospitals in the Netherlands [11], reported that patients admitted on weekends had higher mortality and rebleeding rate than those admitted on weekdays. By contrast, two prospective studies $[14,15]$ conducted in the United Kingdom found no significant difference in mortality of patients with UGIB between weekend and weekday admission groups. A retrospective study conducted in Korea [12], which included 294 cirrhotic patients with acute variceal bleeding, found no significant difference in the in-hospital mortality between weekend and weekday admission groups. Notably, all studies included in the two meta-analyses were not conducted in China mainland. Considering a geographical difference in the staff schedule and management and outcome of AUGIB, further studies should be performed in China mainland.

Second, meta-analyses have also shown that a variation in the source of bleeding leads to different weekend effect on outcomes. All of 4 studies conducted in the United States were based on Nationwide Inpatient Sample, but showed different results $[5,6,8,13]$. The first study demonstrated that patients with UGIB regardless of source of bleeding admitted on weekends had significantly higher mortality and longer length of stay than those admitted on weekdays [5]. The second study also demonstrated that patients with peptic ulcer hemorrhage admitted on weekends had higher mortality and longer length of stay [6]. The third study further confirmed that patients with nonvariceal UGIB admitted on weekends had higher mortality [8]. However, the fourth study found that the mortality in patients with acute variceal bleeding was similar between weekend and weekday admission groups [13].

Third, previous studies usually compared the effect of weekends versus weekdays on the mortality of AUGIB. But the nighttime during weekdays was often ignored from the definition of off-hours. Thus, further studies should refine the interval of off-hours.

Herein, we performed a retrospective study to compare the outcomes of patients with liver cirrhosis and AUGIB who were admitted to a large tertiary hospital of Northeastern China on regular hours versus off-hours.

\section{Methods}

2.1. Study Design. We reviewed the medical records of cirrhotic patients who were consecutively admitted to the General Hospital of Shenyang Military Area from January 2010 to June 2014 as the test cohort. All patients with a diagnosis of liver cirrhosis and AUGIB were eligible. Additionally, we are prospectively collecting all cirrhotic patients who were admitted to our department and underwent contrastenhanced CT scans and endoscopy since December 2014. Thus, based on the data during the patients' enrollment and follow-up, a validation cohort of cirrhotic patients with AUGIB between December 2014 and March 2018 was established for the present study. Age and sex were not limited. The source of bleeding was not limited. Patients with liver and other malignancies were excluded. Patients with incomplete case information and unavailable electronic medical records were also excluded. Data from repeated admission was not deliberately excluded. The outcomes we observed included 5day rebleeding rate, in-hospital mortality, length of hospital stay, and total payment during hospitalizations. This study was approved by the Medical Ethical Committee of our hospital and the ethical approval number was k (2017)42. The patient's informed consent was not required in the retrospective study.

2.2. Data Collection. The primary data collected were age, sex, admission time, etiology of liver disease, and laboratory tests (i.e., red blood cell, hemoglobin, white blood cell, platelet count, total bilirubin, direct bilirubin, indirect bilirubin, albumin, alanine aminotransferase, aspartate aminotransferase, alkaline phosphatase, gamma-glutamyl transpeptidase, blood urea nitrogen, creatinine, potassium, sodium, prothrombin time, activated partial thromboplastin time, and international normalized ratio [INR]). The severity of esophageal varices was also collected. Treatment options of AUGIB were collected as follows: endoscopic therapy (i.e., band ligation, sclerotherapy, and histoacryl), Sengstaken Blackmore tube, somatostatin and/or octreotide, blood transfusion, proton pump inhibitors (PPIs), and surgery.

2.3. Definitions and Formulas. AUGIB was defined as hematemesis and/or melena within 5 days before our admission or positive occult blood test at the day of admission [18]. Regular hours referred to the interval from 8:00 AM to 17:00 $\mathrm{PM}$ at the weekdays (i.e., from Monday to Friday). Otherwise, off-hours were considered, and weekends and public holidays were also considered as off-hours. Child-Pugh score [19] was calculated according to hepatic encephalopathy, ascites, total bilirubin, albumin, and INR. Model for end-stage liver disease $(\mathrm{MELD})$ score $=9.57 \times \ln ($ creatinine $[\mu \mathrm{mol} / \mathrm{L}] \times 0.01)+$ $3.78 \times \ln ($ bilirubin $[\mu \mathrm{mol} / \mathrm{L}] \times 0.05)+11.2 \times \ln (\mathrm{INR})+$ 0.643 [20]. According to the study by Reverter et al. [21], recalibrated MELD score $=-5.312+0.207 \times$ MELD. Albuminbilirubin $(\mathrm{ALBI})$ score $=-0.085 \times$ albumin $(\mathrm{g} / \mathrm{L})+0.66$ $\times \log _{10}$ bilirubin $(\mu \mathrm{mol} / \mathrm{L})[22,23]$.

2.4. Statistical Analyses. Continuous variables were reported as median (range) and were compared using the nonparametric Mann-Whitney U test. Categorical variables were reported as frequency (percentage) and were compared using the chi-square test. Subgroup analyses were also conducted based on the presence of varices on endoscopy (AUGIB with endoscopically confirmed varices and without varices on endoscopy). A 1:1 propensity score matching analysis was performed to adjust the effect of gender, age, Child-Pugh score, MELD score, and recalibrated MELD score on the 
outcomes. A two-tailed $\mathrm{P}<0.05$ was considered statistically significant. All statistical analyses were performed with IBM SPSS 20.0 (IBM Corp.) statistical package and Stata/SE 12.0 (Stata Corp, College Station, TX) software.

\section{Results}

\subsection{Test Cohort}

3.1.1. Patients' Characteristics. Between January 2010 and June 2014, a total of 826 patients with liver cirrhosis and AUGIB were included. Baseline patient characteristics are described in Table 1. Median age was 55.27 years (range: 6.28 to 95.13$)$. Among them, 564 (68.3\%) patients were male. Major etiology of liver diseases included hepatitis B virus infection $(n=208,25.2 \%)$ and alcohol abuse $(n=219,26.5 \%)$. A majority of patients had Child-Pugh class B $(339 / 776,51.4 \%)$. Median MELD score at admission was 6.37 (-7.52 to 38.22). Five hundred and twenty-two patients underwent endoscopy. No, mild, moderate, and severe esophageal varices were observed in 32 (6.1\%), 24 (4.6\%), 54 (10.3\%), and $412(78.9 \%)$ patients, respectively. As for the treatment of AUGIB, 508 (61.5\%) patients underwent endoscopic therapy, $20(2.4 \%)$ patients underwent Sengstaken Blackmore tube placement, $750(90.8 \%)$ patients received somatostatin and/or octreotide, 544 (65.9\%) patients received blood transfusion, 813 (98.4\%) patients received PPIs, and $8(1.0 \%)$ patients underwent surgery. Information regarding 5-day rebleeding was unavailable in 4 patients, because some of their medical records were missing. Five-day rebleeding rate was $14.0 \%$ (115/822). In-hospital mortality was $5.7 \%$ (47/826). Median length of hospital stay was 11.23 days (range: 0.06 to 100.55). Total payment was $¥ 23,120.87$ (range: $1,287.54$ to $226,872.93$ ).

3.1.2. Outcome. Patients admitted on off-hours had lower serum albumin $(\mathrm{P}<0.001)$ and higher white blood cell $(\mathrm{P}<0.001)$, blood urea nitrogen $(\mathrm{P}<0.001)$, potassium $(\mathrm{P}<0.001)$, prothrombin time $(\mathrm{P}=0.034)$, INR $(\mathrm{P}=0.040)$, Child-Pugh score $(\mathrm{P}=0.006)$, and ALBI score $(\mathrm{P}<0.001)$ than those admitted on regular hours (Table 1). As for the interventions, patients admitted on off-hours had a higher proportion of blood transfusion than those admitted on regular hours $(73.3 \%$ versus $60.7 \%, \mathrm{P}<0.001)$. Among the different departments of our hospital, there was no significant difference in the selection of most treatment options for AUGIB between patients admitted on regular hours and off-hours (Supplementary Table 1). As for the outcomes, patients admitted on off-hours had a higher 5-day rebleeding rate $(18.7 \%$ versus $10.7 \%, \mathrm{P}=0.001)$ and a larger amount of payment $(¥ 25,906.83$ versus $¥ 22,017.42, \mathrm{P}<0.001)$. In-hospital mortality was not significantly different between the two groups $(\mathrm{P}=0.418)$. Length of stay was not significantly different between the two groups $(\mathrm{P}=0.830)$.

3.1.3. Subgroup Analyses. The origin of bleeding could be evaluated in 611 patients in the test cohort. They included 591 patients with endoscopically confirmed esophageal and/or gastric varices and 20 patients without varices at endoscopy (Supplementary Table 2).
Among the patients with endoscopically confirmed varices, patients admitted on off-hours were older $(\mathrm{P}=0.015)$ and had lower red blood cell $(\mathrm{P}=0.026)$ and serum albumin $(\mathrm{P}<0.001)$ and higher white blood cell $(\mathrm{P}<0.001)$, blood urea nitrogen $(\mathrm{P}<0.001)$, potassium $(\mathrm{P}=0.001)$, prothrombin time $(\mathrm{P}=0.027)$, INR $(\mathrm{P}=0.04)$, Child-Pugh score $(\mathrm{P}<0.001)$, MELD score $(\mathrm{P}=0.023)$, recalibrated MELD score $(\mathrm{P}=0.023)$, and ALBI score $(\mathrm{P}<0.001)$ than those admitted on regular hours. As for the interventions, patients admitted on offhours had a higher proportion of blood transfusion $(75.5 \%$ versus $59.5 \%, \mathrm{P}<0.001)$ and surgery $(2.1 \%$ versus $0.3 \%$, $\mathrm{P}=0.027)$ than those admitted on regular hours. As for the outcomes, patients admitted on off-hours had a higher 5-day rebleeding rate $(16.5 \%$ versus $10.6 \%, \mathrm{P}=0.038)$ and a larger amount of payment ( $¥ 29,361.51$ versus $¥ 23,864.24, \mathrm{P}<0.001)$. In-hospital mortality and length of stay were not significantly different between the two groups $(\mathrm{P}=0.094$ and $\mathrm{P}=0.856$, respectively).

Among the patients without varices at endoscopy, no significant difference in demographics, etiology of liver disease, laboratory tests, Child-Pugh score, MELD score, recalibrated MELD score, ALBI score, and treatment options was observed between patients admitted on regular hours and off-hours ( $\mathrm{P}>0.05$, in all comparisons). As for the outcomes, none died. Five-day rebleeding rate, length of stay, and total payment were not significantly different between the two groups $(\mathrm{P}=0.117, \mathrm{P}=0.869$, and $\mathrm{P}=0.187$, respectively).

3.1.4. Patients' Characteristics after Propensity Score Matching. After a 1:1 propensity score matching analysis, a total of 226 patients with liver cirrhosis and AUGIB were included. Baseline patient characteristics are described in Table 2. Median age was 54.51 years (range: 6.28 to 81.62). Among them, 144 (63.7\%) patients were male. Major etiology of liver diseases included hepatitis $B$ virus infection $(n=58,25.7 \%)$ and alcohol abuse $(n=50,22.1 \%)$. A majority of patients had Child-Pugh class B $(n=121,53.5 \%)$. Median MELD score at admission was 6.12 (-7.14 to 21.56). No, mild, moderate, and severe esophageal varices were observed in 11 (4.9\%), 19 (4.4\%), 23 (10.2\%), and $182(80.5 \%)$ patients, respectively. As for the treatment of AUGIB, 191 (84.5\%) patients underwent endoscopic therapy, 8 (3.5\%) patients underwent Sengstaken Blackmore tube placement, 218 (96.5\%) patients received somatostatin and/or octreotide, $159(70.4 \%)$ patients received blood transfusion, all patients received PPIs, and 1 (0.4\%) patient underwent surgery. Five-day rebleeding rate was $14.2 \%(n=32)$. In-hospital mortality was $2.2 \%(n=5)$. Median length of hospital stay was 12.835 days (range: 2.76 to 78.00 ). Median total payment was $¥ 28,633.075$ (range: $2,776.55$ to 143,048.30).

3.1.5. Outcomes after Propensity Score Matching. After a 1:1 propensity score matching analysis, 113 patients were matched on each group (Table 2). No significant difference in demographics, etiology of liver disease, laboratory tests, Child-Pugh score, MELD score, recalibrated MELD score, ALBI score, and treatment options was observed between the two groups ( $\mathrm{P}>0.05$, in all comparisons). As for the outcomes, 5 -day rebleeding rate, in-hospital mortality, length of stay, 


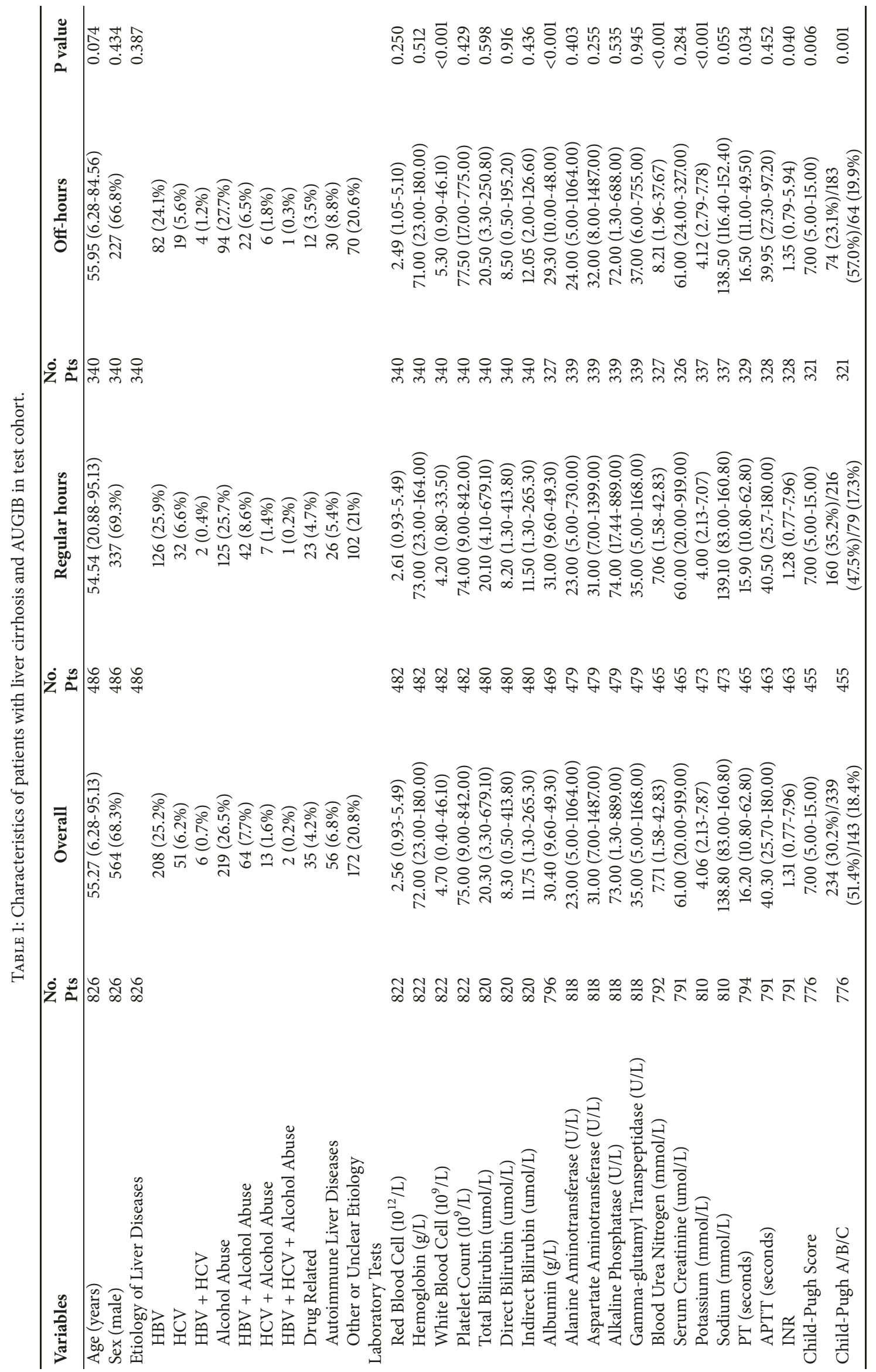




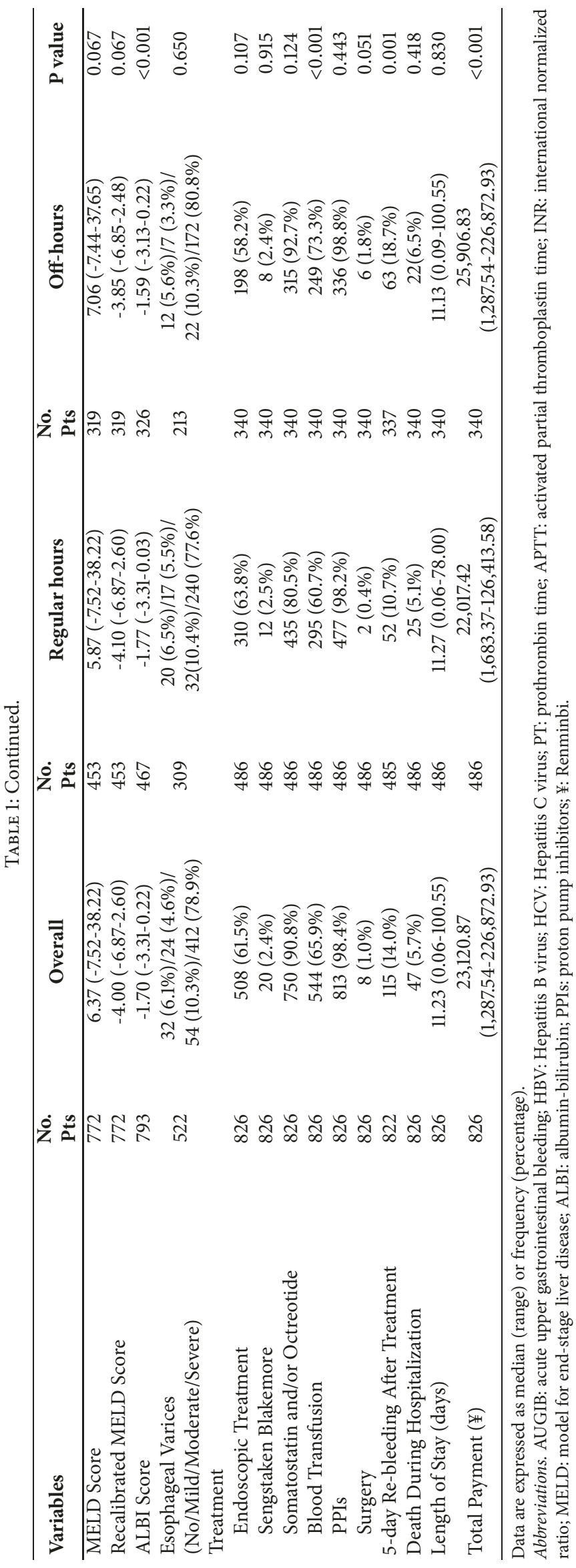




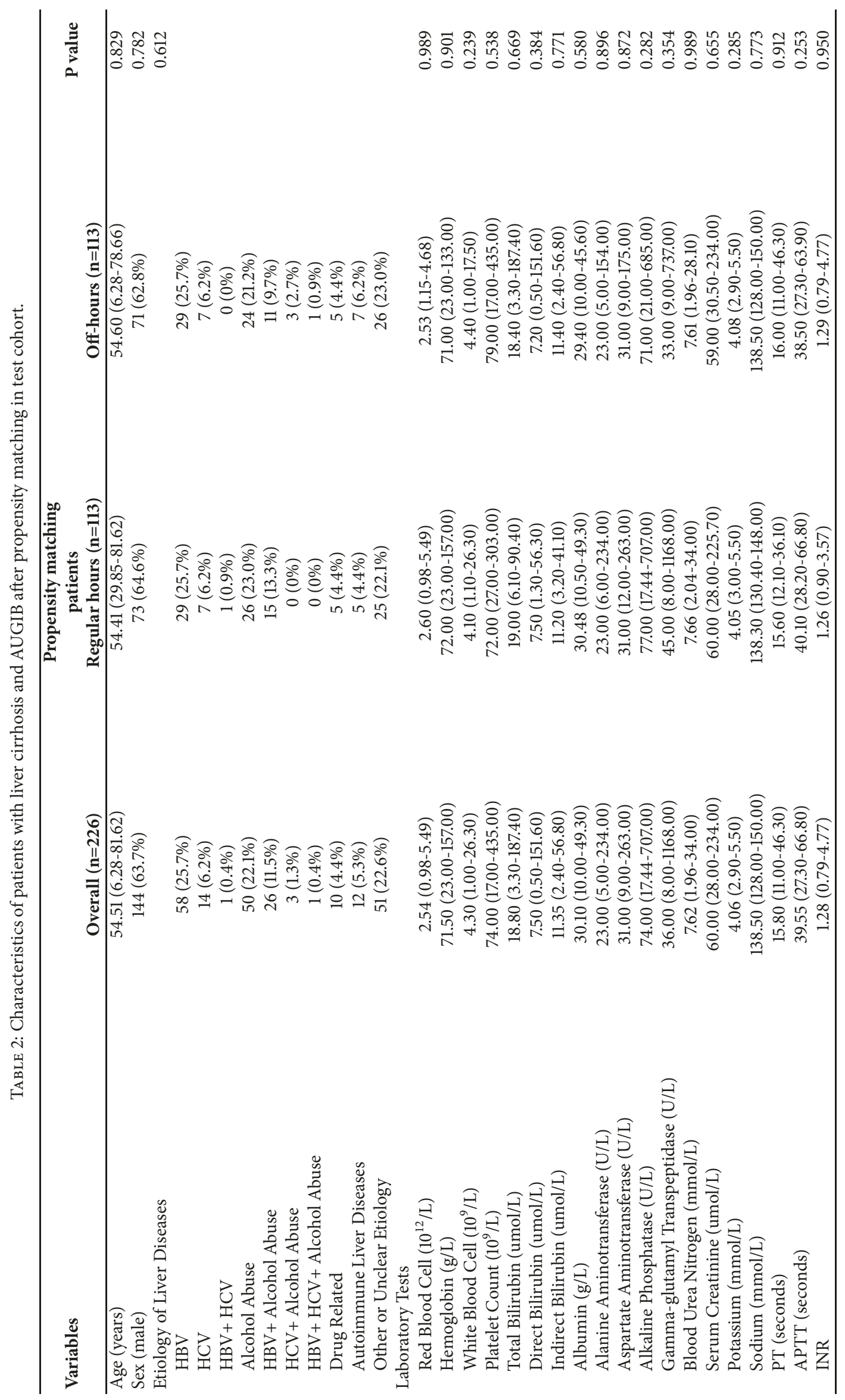




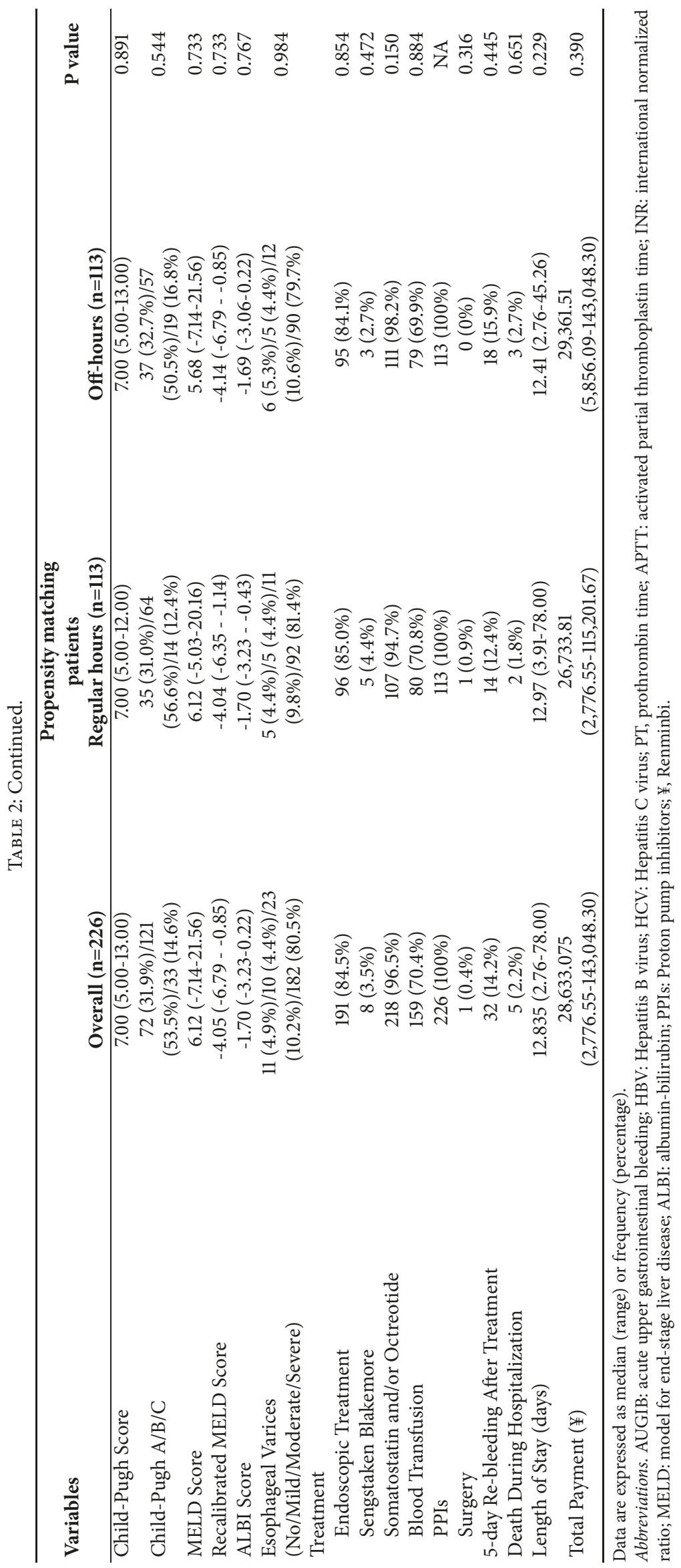


and total payment were not significantly different between the two groups $(\mathrm{P}=0.445, \mathrm{P}=0.651, \mathrm{P}=0.229$, and $\mathrm{P}=0.390$, respectively).

3.1.6. Subgroup Analyses after Propensity Score Matching. After a 1:1 propensity score matching analysis, 140 patients with endoscopically confirmed varices were matched on each group (Supplementary Table 3). Patients admitted on off-hours had higher white blood cell $(\mathrm{P}=0.007)$, blood urea nitrogen $(\mathrm{P}=0.01)$, and potassium $(\mathrm{P}=0.001)$ than those admitted on regular hours. As for the interventions, patients admitted on off-hours had a higher proportion of blood transfusion $(77.1 \%$ versus $58.6 \%, \mathrm{P}=0.001)$ and surgery $(2.9 \%$ versus $0 \%, \mathrm{P}=0.044$ ) than those admitted on regular hours. As for the outcomes, 5-day rebleeding rate, in-hospital mortality, length of stay, and total payment were not significantly different between the two groups $(\mathrm{P}=0.306, \mathrm{P}=0.409, \mathrm{P}=0.421$, and $\mathrm{P}=0.058$, respectively).

\subsection{Validation Cohort}

3.2.1. Patients' Characteristics. Between December 2014 and March 2018, a total of 173 patients with liver cirrhosis and AUGIB were included. Baseline patient characteristics are described in Table 3. Median age was 56.60 years (range: 20.57 to 88.73$)$. Among them, 121 (69.9\%) patients were male. Major etiology of liver diseases included hepatitis B virus infection $(\mathrm{n}=47,27.2 \%)$ and alcohol abuse $(\mathrm{n}=55,31.8 \%)$. A majority of patients had Child-Pugh class B $(94 / 169,55.6 \%)$. Median MELD score at admission was $7.22(-3.16$ to 23.19$)$. One hundred and fifty-one patients underwent endoscopy. No, mild, moderate, and severe esophageal varices were observed in 9 (6.0\%), 23 (15.2\%), 26 (17.2\%), and 93 (61.6\%) patients, respectively. The origin of bleeding could be evaluated in 162 patients, of whom only 4 did not have esophageal and/or gastric varices. As for the treatment of AUGIB, 139 $(80.3 \%)$ patients underwent endoscopic therapy, $1(0.6 \%)$ patients underwent Sengstaken Blackmore tube placement, $160(92.5 \%)$ patients received somatostatin and/or octreotide, 88 (50.9\%) patients received blood transfusion, $173(100 \%)$ patients received PPIs, and $0(0 \%)$ patients underwent surgery. Information regarding 5-day rebleeding was unavailable in one patient. Five-day rebleeding rate was $7.6 \%$ (13/172). In-hospital mortality was $2.3 \%$ (4/173). Median length of hospital stay was 10.10 days (range: 0.12 to 32.94 ). Total payment was $¥ 24,328.31$ (range: $3,427.24$ to $98,215.78$ ).

3.2.2. Outcomes. No significant difference in demographics, etiology of liver disease, laboratory tests, Child-Pugh score, MELD score, recalibrated MELD score, ALBI score, and treatment options was observed between the two groups ( $\mathrm{P}>0.05$, in all comparisons). As for the outcomes, 5-day rebleeding rate, in-hospital mortality, length of stay, and total payment were not significantly different between the two groups $(\mathrm{P}=0.579, \mathrm{P}=0.973, \mathrm{P}=0.335$, and $\mathrm{P}=0.166$, respectively) (Table 3 ).

3.2.3. Patients' Characteristics after Propensity Score Matching. After a 1:1 propensity score matching analysis, a total of
40 patients with liver cirrhosis and AUGIB were included. Baseline patient characteristics are described in Table 4. Median age was 56.86 years (range: 20.57 to 75.64 ). Among them, 28 (70\%) patients were male. Major etiology of liver diseases included hepatitis B virus infection $(n=9,22.5 \%)$ and alcohol abuse $(n=14,35 \%)$. A majority of patients had ChildPugh class B $(n=22,55 \%)$. Median MELD score at admission was 5.82 (range: -2.38 to 23.19 ). No, mild, moderate, and severe esophageal varices were observed in $2(5 \%), 5(12.5 \%)$, $6(15 \%)$, and $27(67.5 \%)$ patients, respectively. As for the treatment of AUGIB, 38 (95\%) patients underwent endoscopic therapy, no patient underwent Sengstaken Blackmore tube placement, 39 (97.5\%) patients received somatostatin and/or octreotide, 21 (52.5\%) patients received blood transfusion, all patients received PPIs, and no patient underwent surgery. Five-day rebleeding rate was $12.5 \%(n=5)$. In-hospital mortality was $2.5 \%(n=1)$. Median length of hospital stay was 11.95 days (range: 5.73 to 31.06 ). Median total payment was $¥ 24,961.33$ (range: $11,212.15$ to $81,125.52$ ).

3.2.4. Outcomes after Propensity Score Matching. After a 1:1 propensity score matching analysis, 20 patients were matched on each group (Table 4). No significant difference in demographics, etiology of liver disease, laboratory tests, Child-Pugh score, MELD score, recalibrated MELD score, ALBI score, and treatment options was observed between the two groups ( $\mathrm{P}>0.05$, in all comparisons). As for the outcomes, 5-day rebleeding rate, in-hospital mortality, length of stay, and total payment were not significantly different between the two groups $(\mathrm{P}=0.633, \mathrm{P}=0.311, \mathrm{P}=0.441$, and $\mathrm{P}=0.829$, respectively).

\section{Discussion}

Traditionally, a worse outcome in patients with UGIB during the weekend was potentially attributed to lower staffing levels and relatively younger and inexperienced staff [11]. Indeed, both overall analyses and subgroup analyses of patients with endoscopically confirmed varices in the test cohort demonstrated a significantly higher 5-day rebleeding rate and a larger amount of payment in patients admitted on off-hours. This might be primarily due to worse liver dysfunction in patients admitted on off-hours, such as lower albumin and higher prothrombin time, INR, Child-Pugh score, and ALBI score. As the Child-Pugh score was matched, the propensity score matching analyses of both test and validation cohorts showed no significant effect of admission time on the rebleeding rate, in-hospital mortality, length of stay, and total payment of cirrhotic patients with AUGIB. These findings suggested that the weekend effect might not be an independent risk factor for worse outcomes of cirrhotic patients with AUGIB.

A meta-analysis [16] found that off-hours admission was not associated with a higher risk of rebleeding rate $(\mathrm{OR}=1.06,95 \% \mathrm{CI}=0.83-1.35$, and $\mathrm{P}=0.66)$ and longer length of stay (WMD 0.06 day, $95 \% \mathrm{CI}=-0.30--0.42, \mathrm{P}=0.747$ ). These previous findings were consistent with our results regarding 5 -day rebleeding rate and length of stay. Notably, our study focused on the 5-day rebleeding rate after treatment during 


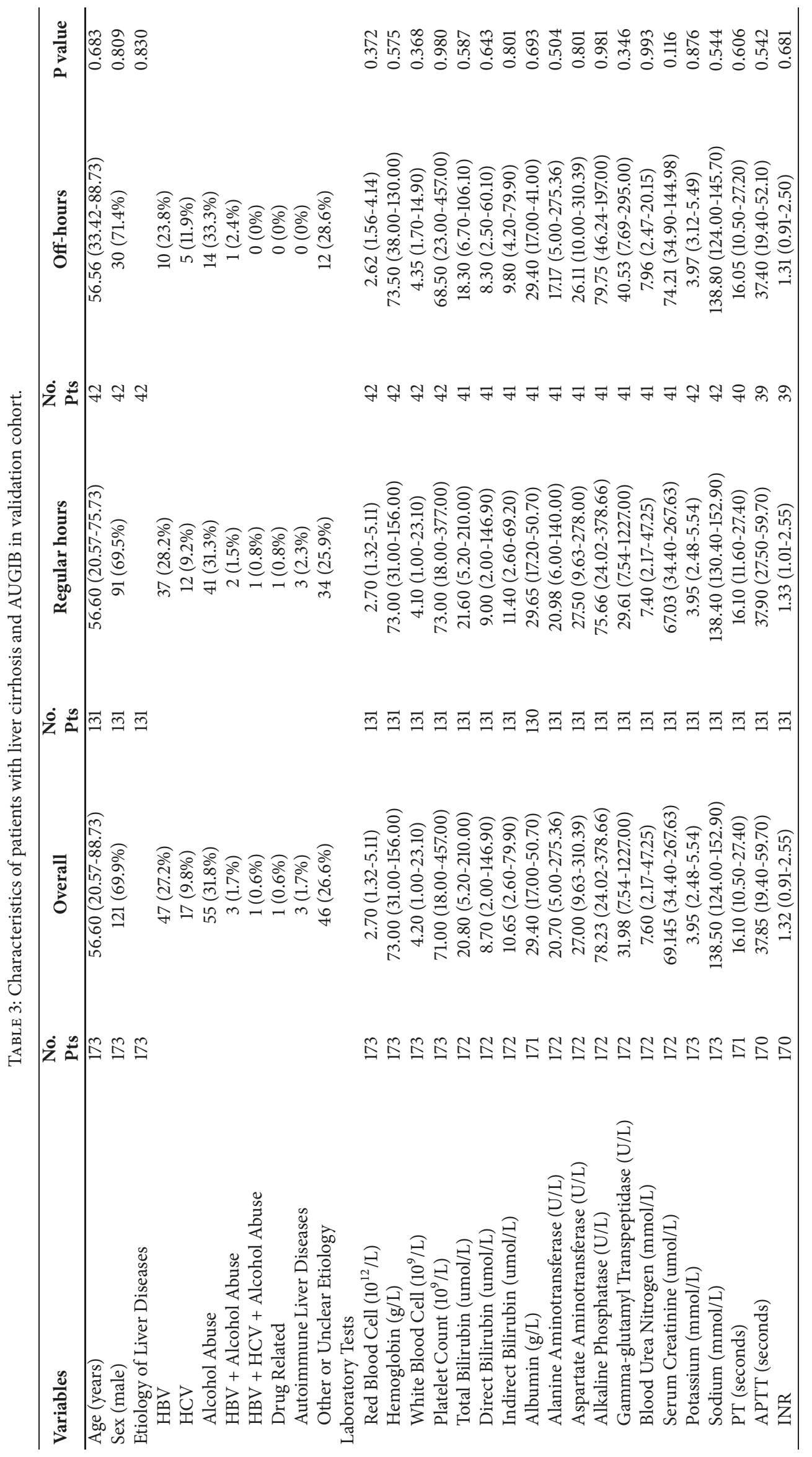




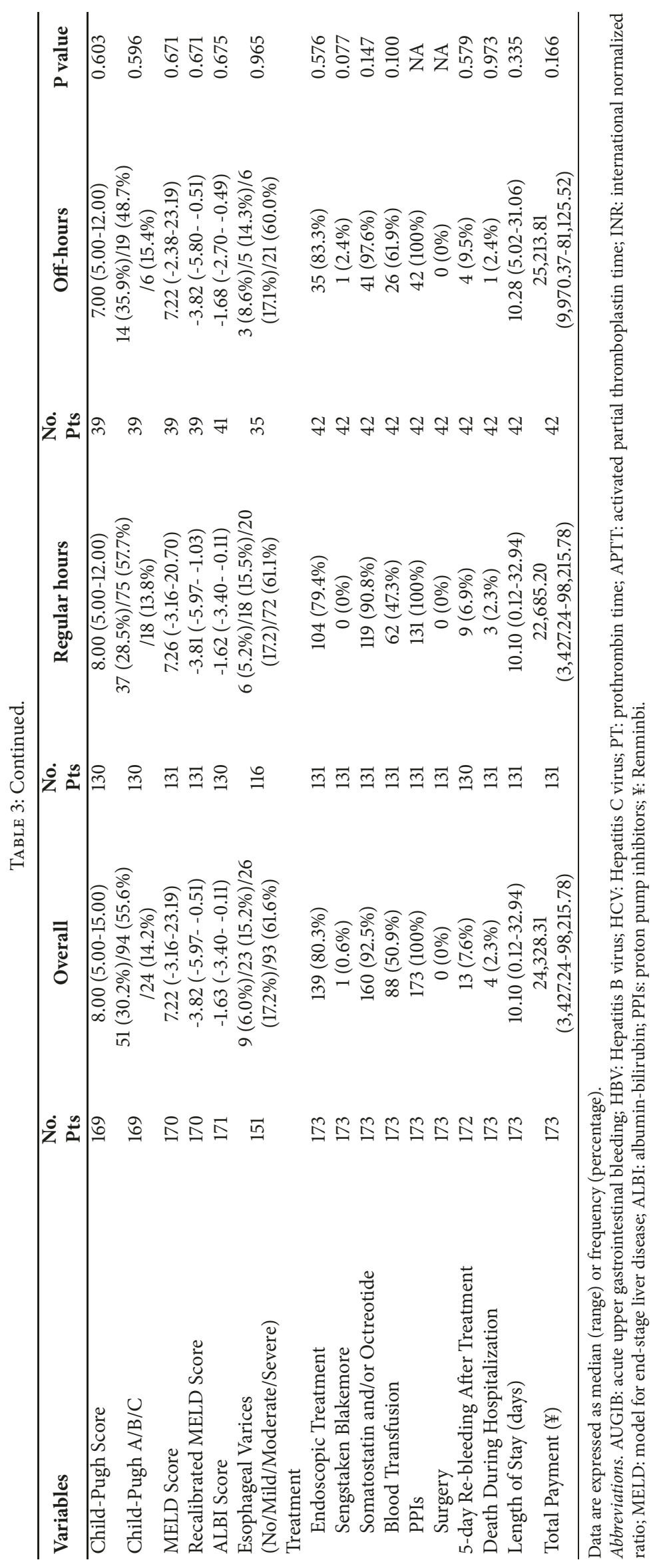




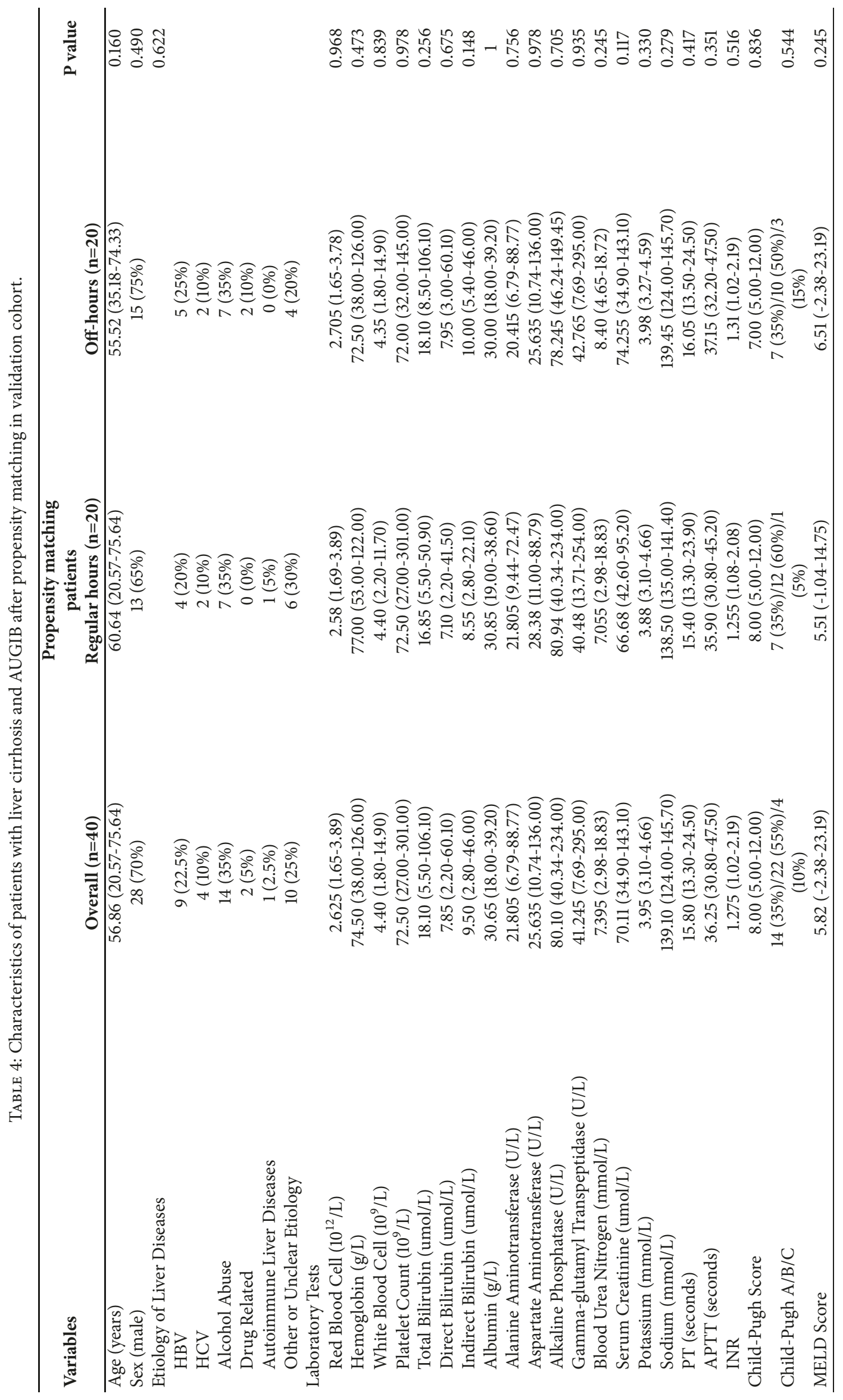




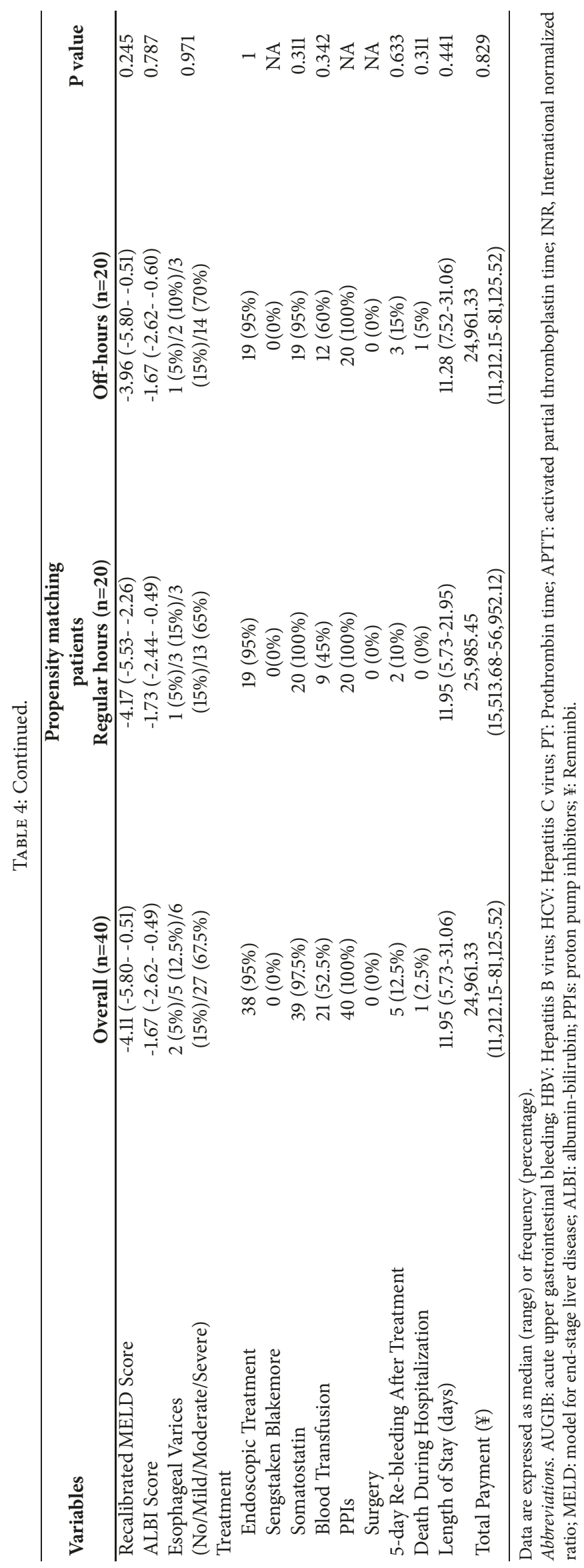


hospitalization. However, the interval of rebleeding was not specified in the meta-analysis. As we have known, the length of stay was usually associated with the severity of illness [24]. In addition, as well known, liver dysfunction as estimated by Child-Pugh score and MELD score were risk factors for mortality of cirrhotic patients with AUGIB [25-27].

Two of the published meta-analyses $[16,17]$ demonstrated a significant weekend effect on the mortality in patients with nonvariceal UGIB, but not those with variceal bleeding. This finding seemed to be consistent with our results regarding mortality. Notably, our study focused on the outcomes during hospitalization, but not those after discharge. The fact is readily understood that admission time mainly influenced the in-hospitalization outcomes but marginally influenced the outcomes after discharge. Indeed, a meta-analysis also suggested that off-hours admission negatively influenced inhospital mortality $(\mathrm{P}=0.009)$, rather than 30 -day mortality $(\mathrm{P}=0.116)$.

Our study has the following advantages. First, we refined the definitions of off-hours admission. Second, we included a test cohort and a validation cohort which can reduce the sampling bias to some extent. Third, we employed a propensity score matching analysis. Thus, the patient characteristics, especially Child-Pugh and MELD scores which are significantly associated with prognosis of liver cirrhosis, are comparable between the two groups. Our results become more stable. However, the major drawback of our study should be that not all patients undergo endoscopy to evaluate the source of bleeding. Additionally, the sample size is not adequate in the validation cohort. Finally, a potential selection bias could not be neglected due to the retrospective nature of this study.

In conclusion, off-hours admission might not be associated with outcomes in patients with liver cirrhosis and AUGIB. However, the geographical difference should not be neglected to extrapolate our findings.

\section{Abbreviations \\ AUGIB: Acute upper gastrointestinal bleeding \\ UGIB: Upper gastrointestinal bleeding \\ INR: International normalized ratio \\ PPIs: $\quad$ Proton pump inhibitors \\ MELD: Model for end-stage liver disease \\ ALBI: Albumin-bilirubin.}

\section{Data Availability}

The data used to support the findings of this study are available from the corresponding author upon request.

\section{Disclosure}

Yingying Li and Bing Han are co-first authors.

\section{Conflicts of Interest}

The authors declare that they have no conflicts of interest.

\section{Authors' Contributions}

Yingying Li reviewed and searched the literature, wrote the protocol, collected the data, performed the statistical analysis, interpreted the data, and drafted the manuscript. Bing Han and Tingxue Song performed the statistical analysis and critical revision of the manuscript for important intellectual content. Wenchun Bao, Ran Wang, Zhaohui Bai, Kexin Zheng, and Qianqian Li collected the data and revised the manuscript. Hongyu Li and Xiaozhong Guo checked the data and gave critical comments. Xingshun Qi conceived the work, wrote the protocol, performed the statistical analysis, interpreted the data, and revised the manuscript. All authors have made an intellectual contribution to the manuscript and approved the submission. This work was partially presented at the 18th Congress of Gastroenterology China that was held in Dalian, China on September 2018.

\section{Acknowledgments}

This study was partially supported by the grant from the Fourth Batch of Science and Technology Planning Projects of Liaoning Province in 2015 (no. 2015225019) for Professor Xiaozhong Guo.

\section{Supplementary Materials}

Supplementary Table 1 . Treatment of cirrhotic patients with AUGIB at different departments from our hospital in test cohort. Supplementary Table 2. Subgroup analyses of patients with liver cirrhosis and AUGIB based on the origin of bleeding in test cohort. Supplementary Table 3. AUGIB patients with endoscopically confirmed varices after propensity matching analysis in test cohort. (Supplementary Materials)

\section{References}

[1] J. P. Hreinsson, E. Kalaitzakis, S. Gudmundsson, and E. S. Björnsson, "Upper gastrointestinal bleeding: Incidence, etiology and outcomes in a population-based setting," Scandinavian Journal of Gastroenterology, vol. 48, no. 4, pp. 439-447, 2013.

[2] L. A. Button, S. E. Roberts, P. A. Evans et al., "Hospitalized incidence and case fatality for upper gastrointestinal bleeding from 1999 to 2007: a record linkage study," Alimentary Pharmacology \& Therapeutics, vol. 33, no. 1, pp. 64-76, 2011.

[3] I. M. Gralnek, "Evaluating the "weekend effect" on patient outcomes in upper GI bleeding," Gastrointestinal Endoscopy, vol. 80, no. 2, pp. 236-238, 2014.

[4] D. J. Shulkin, "Like night and day - Shedding light on off-hours care," The New England Journal of Medicine, vol. 358, no. 20, pp. 2091-2093, 2008.

[5] S. D. Dorn, N. D. Shah, B. P. Berg, and J. M. Naessens, "Effect of weekend hospital admission on gastrointestinal hemorrhage outcomes," Digestive Diseases and Sciences, vol. 55, no. 6, pp. 1658-1666, 2010.

[6] A. A. M. Shaheen, G. G. Kaplan, and R. P. Myers, "Weekend versus weekday admission and mortality from gastrointestinal hemorrhage caused bypeptic ulcer disease," Clinical Gastroenterology and Hepatology, vol. 7, no. 3, pp. 303-310, 2009. 
[7] A. Ahmed, M. Armstrong, I. Robertson, A. J. Morris, O. Blatchford, and A. J. Stanley, "Upper gastrointestinal bleeding in Scotland 2000-2010: Improved outcomes but a significant weekend effect," World Journal of Gastroenterology, vol. 21, no. 38, pp. 10890-10897, 2015.

[8] A. N. Ananthakrishnan, E. L. McGinley, and K. Saeian, "Outcomes of weekend admissions for upper gastrointestinal hemorrhage: a nationwide analysis," Clinical Gastroenterology and Hepatology, vol. 7, no. 3, pp. 296-302, 2009.

[9] C. M. Bell and D. A. Redelmeier, "Mortality among patients admitted to hospitals on weekends as compared with weekdays," The New England Journal of Medicine, vol. 345, no. 9, pp. 663668, 2001.

[10] J. M. Haas, J. D. Gundrum, and S. W. Rathgaber, "Comparison of time to endoscopy and outcome between weekend/weekday hospital admissions in patients with upper GI hemorrhage," Wisconsin Medical Journal, vol. 111, no. 4, pp. 161-165, 2012.

[11] N. L. De Groot, J. H. Bosman, P. D. Siersema, M. G. H. Van Oijen, and A. J. Bredenoord, "Admission time is associated with outcome of upper gastrointestinal bleeding: Results of a multicentre prospective cohort study," Alimentary Pharmacology \& Therapeutics, vol. 36, no. 5, pp. 477-484, 2012.

[12] S. J. Byun, S. U. Kim, J. Y. Park et al., "Acute variceal hemorrhage in patients with liver cirrhosis: Weekend versus weekday admissions," Yonsei Medical Journal, vol. 53, no. 2, pp. 318-327, 2012.

[13] P. P. Myers, G. G. Kaplan, and A. M. Shaheen, "The Effect of Weekend versus Weekday Admission on Outcomes of Esophageal Variceal Hemorrhage," Canadian Journal of Gastroenterology \& Hepatology, vol. 23, Article ID 713789, pp. 495$501,2009$.

[14] V. Jairath, B. C. Kahan, R. F. A. Logan et al., "Mortality from acute upper gastrointestinal bleeding in the United Kingdom: does it display a "weekend effect"?" American Journal of Gastroenterology, vol. 106, no. 9, pp. 1621-1628, 2011.

[15] I. A. Murray, H. R. Dalton, A. J. Stanley et al., "International prospective observational study of upper gastrointestinal haemorrhage: Does weekend admission affect outcome?" United European Gastroenterology Journal, vol. 5, no. 8, pp. 1082-1089, 2017.

[16] X. F. Xia, P. W. Y. Chiu, K. K. F. Tsoi, F. K. L. Chan, J. J. Y. Sung, and J. Y. W. Lau, "The effect of off-hours hospital admission on mortality and clinical outcomes for patients with upper gastrointestinal hemorrhage: A systematic review and meta-analysis of 20 cohorts," United European Gastroenterology Journal, vol. 6, no. 3, pp. 367-381, 2018.

[17] A. Gupta, R. Agarwal, and A. N. Ananthakrishnan, “"Weekend Effect” in Patients With Upper Gastrointestinal Hemorrhage: A Systematic Review and Meta-analysis," American Journal of Gastroenterology, vol. 113, no. 1, pp. 13-21, 2018.

[18] R. de Franchis, "Revising consensus in portal hypertension: report of the Baveno $\mathrm{V}$ consensus workshop on methodology of diagnosis and therapy in portal hypertension," Journal of Hepatology, vol. 53, no. 4, pp. 762-768, 2010.

[19] R. N. H. Pugh, I. M. Murray-Lyon, J. L. Dawson, M. C. Pietroni, and R. Williams, "Transection of the oesophagus for bleeding oesophageal varices," British Journal of Surgery, vol. 60, no. 8, pp. 646-649, 1973.

[20] P. S. Kamath and W. R. Kim, "The model for end-stage liver disease (MELD)," Hepatology, vol. 45, no. 3, pp. 797-805, 2007.

[21] E. Reverter, P. Tandon, S. Augustin et al., "A MELD-based model to determine risk of mortality among patients with acute variceal bleeding," Gastroenterology, vol. 146, no. 2, pp. 412-e3, 2014.

[22] P. J. Johnson, S. Berhane, C. Kagebayashi et al., "Assessment of Liver Function in Patients With Hepatocellular Carcinoma: A New Evidence-Based Approach-The ALBI Grade," Journal of Clinical Oncology, vol. 33, no. 6, pp. 550-558, 2015.

[23] D. Zou, X. Qi, C. Zhu et al., "Albumin-bilirubin score for predicting the in-hospital mortality of acute upper gastrointestinal bleeding in liver cirrhosis: A retrospective study," The Turkish Journal of Gastroenterology, vol. 27, no. 2, pp. 180-186, 2016.

[24] Y. H. Youn, Y. J. Park, J. H. Kim, T. J. Jeon, J. H. Cho, and H. Park, "Weekend and nighttime effect on the prognosis of peptic ulcer bleeding," World Journal of Gastroenterology, vol. 18, no. 27, pp. 3578-3584, 2012.

[25] K. Bambha, W. R. Kim, R. Pedersen, J. P. Bida, W. K. Kremers, and P. S. Kamath, "Predictors of early re-bleeding and mortality after acute variceal haemorrhage in patients with cirrhosis," Gut, vol. 57, no. 6, pp. 814-820, 2008.

[26] Y. Peng, X. Qi, and X. Guo, "Child-Pugh Versus MELD Score for the Assessment of Prognosis in Liver Cirrhosis," Medicine, vol. 95, no. 8, Article ID e2877, 2016.

[27] Y. Peng, X. Qi, J. Dai, H. Li, and X. Guo, "Child-Pugh versus MELD score for predicting the in-hospital mortality of acute upper gastrointestinal bleeding in liver cirrhosis," International Journal of Clinical and Experimental Medicine, vol. 8, pp. 751757, 2015. 


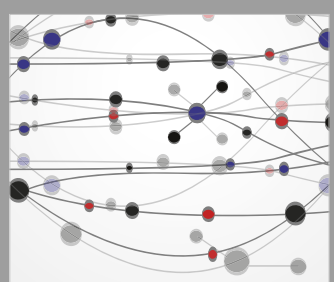

The Scientific World Journal
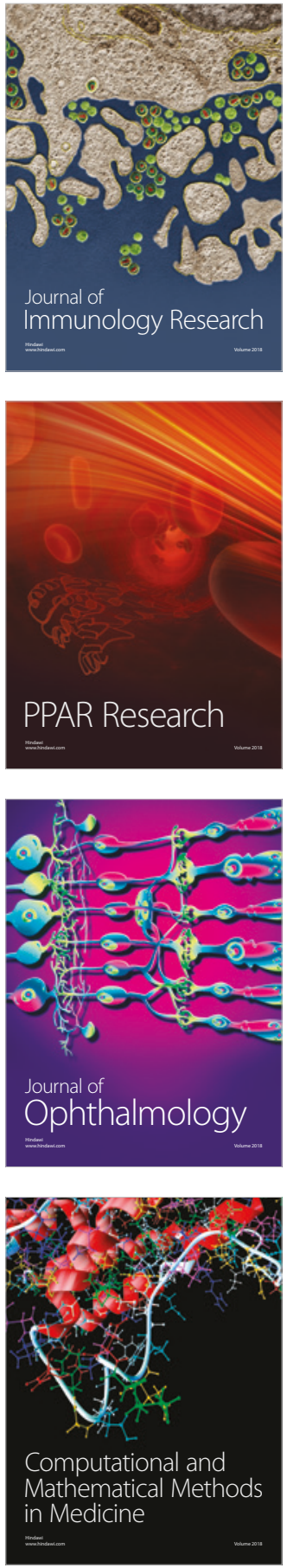

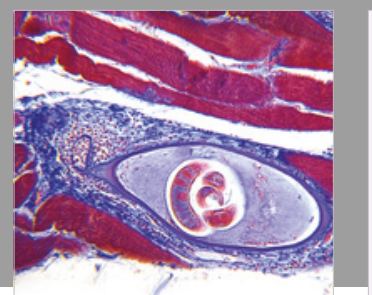

Gastroenterology Research and Practice

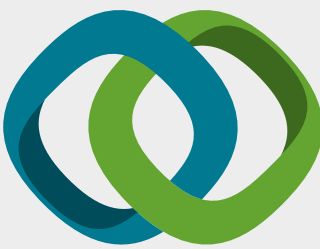

\section{Hindawi}

Submit your manuscripts at

www.hindawi.com
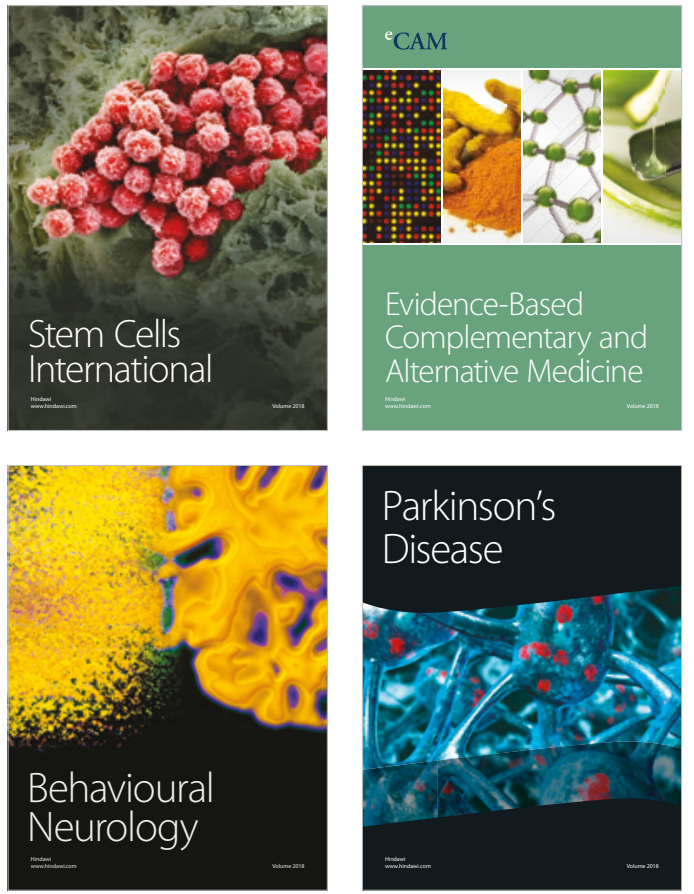

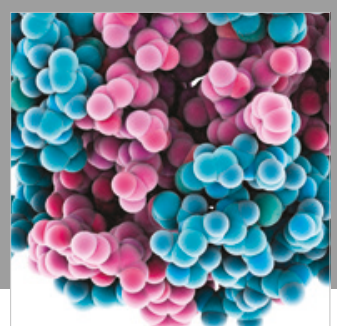

ournal of

Diabetes Research

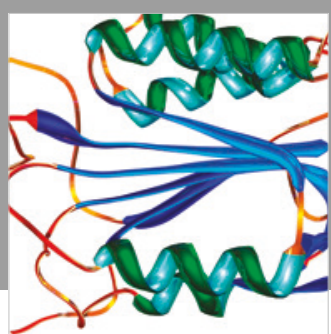

Disease Markers
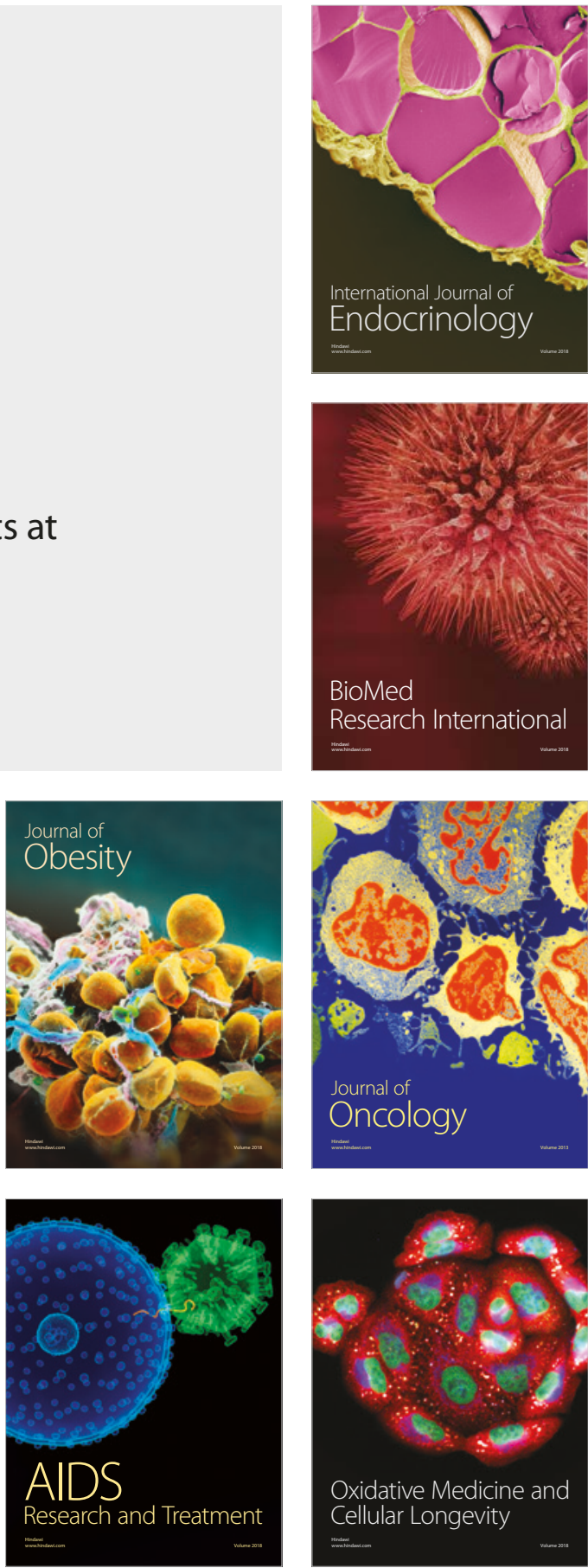